\title{
Aspectos cualitativos del aprendizaje en el Programa de Ética de la Investigación Biomédica y Psicosocial del Centro Interdisciplinario de Estudios en Bioética de la Universidad de Chile
}

Eduardo Rodríguez ${ }^{2}$

Resumen: El presente trabajo reflexiona sobre la experiencia de 10 años en procesos de aprendizaje grupal y adquisición de competencias, de los participantes en el Programa de Ética de la Investigación Biomédica y Psicosocial del Centro Interdisciplinario de Estudios en Bioética de la Universidad de Chile, Fogarty Grant R25TW6056. Considera el papel de la bioética en la formación en su carácter transdisciplinario.

Palabras clave: ética de investigación, formación, bioética

Learning qualitative aspects of the ethics of biomedical and psychosocial research program of the Interdisciplinary Center for Studies on Bioethics, University of Chile

Abstract: This article reflects on the ten years experience of group learning processes and skills acquisition by trainees of the ethics of biomedical and psychosocial research international training program from the Interdisciplinary Center for Studies on Bioethics of the University of Chile, Fogarty Grant R25TW6056. It takes into consideration the role of bioethics in training in its transdisciplinary character.

Key words: ethics of research, training, bioethics

Aspectos qualitativos da aprendizagem no Programa de Ética da Pesquisa Biomédica e Psicossocial do Centro Interdisciplinar de Estudos em Bioética da Universidade do Chile

Resumo: O presente trabalho reflete a experiência de 10 anos em processos de aprendizagem em grupo e aquisição de competências dos participantes no Programa de Ética da Pesquisa Biomédica e Psicossocial do Centro Interdisciplinar de Estudos em Bioética da Universidade do Chile, Fogarty Grant R25TW6056. Considera o papel da bioética na formação de seu caráter transdisciplinar.

Palavras-chave: ética da pesquisa, formação, bioética

\footnotetext{
${ }^{1}$ El artículo ha sido realizado sobre la base de la experiencia de egresados del Programa de Ética de Investigación Biomédica y Psicosocial del Centro Interdisciplinario de Estudios en Bioética de la Universidad de Chile, Fogarty Grant R25TW6056.

${ }^{2}$ Coordinador del Programa de Ética de Investigación Biomédica y Psicosocial, Centro Interdisciplinario de Estudios en Bioética, Universidad de Chile, Chile

Correspondencia: erodriguezchi@gmail.com
} 


\section{Introducción}

El Programa Internacional de Ética de la Investigación Biomédica y Psicosocial del Centro Interdisciplinario de Estudios en Bioética de la Universidad de Chile, Fogarty Grant R25TW6056, por el que han pasado 50 profesionales(1), cuenta con 10 años de experiencia en el entrenamiento de profesionales de países latinoamericanos. El programa capacita para asumir un liderazgo activo en la enseñanza, evaluación, diseño y conducción de investigaciones éticamente sustentables, colaborar en normativas y regulaciones de ética de la investigación, así como para participar en comités de evaluación ética de la investigación científica, con el compromiso de apoyar y crear estos campos dentro de su institución, retribuyendo en servicios lo que se recibe en formación.

Se trabaja con pequeños grupos de profesionales de alrededor de cinco miembros por año. Uno de los frutos de esta forma de trabajo ha sido reflexionar sobre procesos de aprendizaje y adquirir competencias, lo que se refleja en el presente artículo. Dejar a un lado el trabajo regular gracias al apoyo de una beca permite concentrarse en la reflexión sobre temas éticos y en cómo superar obstáculos y encontrar estrategias para mejorar los sistemas de control ético en los distintos países e instituciones. Integrar una forma de hacer ética en el complejo mundo académico y de la investigación exige competencias personales y profesionales apropiadas a cada diferente contexto, teniendo en consideración que existen numerosos obstáculos administrativos y estructurales en la mayoría de los contextos en Latinoamérica.

\section{Objetivos del Programa}

- Ofrecer una formación interdisciplinaria en ética de la investigación biomédica y psicosocial (papel activo en investigación y en su impacto social).

- Reforzar la capacidad de los países latinoamericanos para abordar los aspectos éticos y sociales de la investigación científica en el contexto de la investigación internacional.

- Aumentar el nivel de conocimiento e infor- mación mediante la promoción de la investigación en bioética y la generación de capacidades de producción científica en los ámbitos local, regional y nacional.

- Reforzar las habilidades analíticas, fortaleciendo la autonomía intelectual, la capacidad crítica y la intención de publicar en revistas científicas y de formular regulaciones.

- Desarrollar competencias y actitudes de mediación y negociación para resolver dilemas bioéticos y toma de decisiones en forma transdisciplinaria.

- Desarrollar componentes curriculares en el ámbito de magíster, recursos pedagógicos y planes de evaluación.

- Mejorar las habilidades para interactuar con los formuladores de políticas públicas.

- Desarrollar el trabajo interdisciplinario y adquirir habilidades relevantes para participar en comités de ética de investigación y asesoría.

- Trabajar en red en ética de la investigación en la región.

\section{Experiencia de aprendizaje grupal}

- Los candidatos profesionales que participan se seleccionan preferentemente de acuerdo con los siguientes criterios:

- Grado académico avanzado en instituciones acreditadas (M.D., Ph.D, Magíster).

- Experiencia de participación previa en comités de ética de investigación o en planificación/regulación de atención en salud.

- Antecedentes de haber investigado con demostrada competencia mediante publicaciones.

- Conocimiento avanzado del idioma inglés.

- Plan de trabajo posterior con apoyo institucional.

A pesar de la selección, con frecuencia los profesionales que han participado difieren en sus conocimientos sobre metodología de investigación, bioética y habilidad de publicar, por lo que buena parte del tiempo se dedica a procesos de nivelación. Esto debido a que una buena parte de los que postulan no alcanzan el perfil solicitado. Por 
lo general, ha habido dificultad en motivar a participar o en encontrar a investigadores de trayectoria que tengan conocimientos de bioética, sobre todo en algunos de los países de la región.

Se busca que cada promoción conforme un grupo con suficiente variedad profesional para potenciar el trabajo interdisciplinario. En estos diez años se ha trabajado con grupos pequeños, de cuatro a ocho profesionales, con trabajo presencial de nueve meses - al inicio del programa- y, en los últimos años, de tres meses. También el profesorado es interdisciplinario y se busca el diálogo horizontal. Trabajar con pequeños grupos de profesionales de distintos países constituye una buena condición para el diálogo fructífero y el intercambio de información, facilitando la posterior formación de redes.

La aproximación del programa al aprendizaje descansa en la convicción de que las potencialidades personales pueden actualizarse mediante la guía del profesor y la interacción con otros profesionales(2); el protagonista no es el profesor que transmite el conocimiento, sino el alumno, en este caso un profesional que desarrolla habilidades de liderazgo, adquiere actitudes éticas, desarrolla habilidades comunicativas y de resolución de problemas y aprende a diseñar y evaluar éticamente la investigación. El papel del profesor no es solamente transmitir conocimiento, sino aconsejar en la toma de decisiones éticas y facilitar el aprendizaje mediante dinámicas grupales y la promoción de trabajo colaborativo(3). Además, se integra la tutoría para aprender metodología y diseño de investigación, y la evaluación ética y científica de investigaciones.

Como actitud profesional, se considera importante que los profesores traten a los participantes del programa como colegas. Se elige al profesor en relación con su conocimiento en el campo de estudio y sus actitudes éticas de tolerancia, creatividad, flexibilidad, compromiso con la ética profesional y respeto para generar un buen clima de aprendizaje.

En el aprendizaje, es necesario no solo aprender bien todo conocimiento teórico exigido - como la aplicación de principios éticos, regulaciones y pautas éticas internacionales en ética de investigación-, sino también desarrollar habilidades mediante experiencias prácticas, generalmente muy apreciadas por los participantes. En el programa, los alumnos tienen la oportunidad de simular sesiones de evaluación de protocolos de investigación, como se realizan en los comités de ética; discutir resolución de casos que ejemplifican la aplicación de un principio ético o una falta de integridad ética; participar en sesiones de comités de ética de investigación cuando se decide aprobar o rechazar un determinado protocolo; trabajar como grupo interdisciplinario en la redacción de una propuesta de grant para una agencia internacional; enseñar conceptos y aplicaciones de ética de investigación en pregrado y posgrado; diseñar intervenciones concretas institucionales (formar o fortalecer comités de ética, introducir currículos en ética de investigación en instituciones académicas, proponer regulaciones y normativas en ética de investigación, desarrollar redes y sitios web en ética de investigación); participar con investigadores cuando realizan trabajo de campo; formar parte de redes. La participación en conferencias, actividades de enseñanza y el servicio a instituciones y organismos gubernamentales, como los ministerios de salud en los distintos países, ha sido un método útil para mantener activa la red.

En las sesiones de discusión de casos ha sido importante seguir una estructura predeterminada para presentarlos y debatir en profundidad las posibles respuestas a las consecuencias expuestas por el caso, siguiendo el método deliberativo de argumentación en bioética. Conocer de dinámica grupal, para evitar que la discusión sea acaparada por alguno de los participantes, con poca participación del resto, es también importante. El análisis de casos ayuda a desarrollar el pensamiento crítico, el análisis contextual y las habilidades de resolución de problemas y de comunicación. Se sigue la siguiente estructura:

- Lectura del caso.

- Análisis de los hechos.

- Identificación de problemas y de principios bioéticos afectados.

- Propuesta de soluciones.

- Consenso. 
Un aspecto relevante del programa es trabajar en el diseño e implementación de investigaciones en bioética. Enseñar cómo investigar en bioética es una tarea compleja, especialmente cuando los participantes tienen diferentes experiencias profesionales y grado de conocimiento de metodología de investigación. La aproximación del programa ha sido enseñar metodología cuantitativa y cualitativa para nivelar conocimientos y aprender a diseñar investigación con la ayuda de un tutor y la interacción con los demás colegas. El papel de los tutores es guiar a los participantes en el proceso de diseño de la investigación y de escritura de los resultados(4).

\section{Papel de la bioética}

En el aprendizaje grupal con trabajo interdisciplinario se toma como base común la disciplina de la bioética, bajo cuyos conceptos se interpretan los problemas presentados por el profesor, que tiene la función de ayudar al proceso comunicativo y el intercambio de puntos de vista, de forma que los profesionales puedan aprender interactivamente de sus experiencias personales. Se proporciona conocimiento sobre cómo argumentar y deliberar y sobre diferentes escuelas de pensamiento que han abordado la argumentación con base filosófica en temas de ética de investigación. Aprender las rela- ciones entre teorías éticas ayuda a argumentar con fundamentación filosófica(5). Se busca identificar y diferenciar interacciones e intersecciones entre bioética y variables sociales y culturales, en conjunto con la reflexión filosófica.

De esta forma, se llega a entender la forma de resolver éticamente problemas relacionados con la práctica de investigación y especificar las acciones que se requieren. La bioética tiene la capacidad de ser transdisciplinaria, al hacer emerger nuevo conocimiento transcendiendo los campos del saber tradicional(6). El Programa ha seguido la práctica de aprender a argumentar de acuerdo con la teoría del diálogo cuando existen diferentes puntos de vista respecto a una situación o problema. Siguiendo a Habermas(7), todos los argumentos deben atenderse respetando que somos personas autónomas, pero se requiere consenso en la forma de presentar los argumentos, de manera que podamos entendernos en un campo común, separado de condicionamientos culturales y educacionales. En la ética comunicativa, los intereses particulares de los agentes, lejos de ser sometidos a un proceso de intercambio, son sometidos a un proceso de universalización, y los interlocutores se enriquecen en la relación y respetan sus respectivos valores.

Tabla 1. Contribuciones de los participantes a la ética de investigación.

\begin{tabular}{|l|l|}
\hline Contribuciones participantes (total: 50) & Posiciones después del entrenamiento \\
\hline - Proyectos de Investigación: $64(50)^{*}$ & Profesor Universidad (Docencia ética de investigación): 33 \\
- Publicaciones & Instituto Nacional de Salud: 10 \\
Artículos: $54(21)^{*}$ & Instituciones de Investigación: 5 \\
Capítulos de libros: $52(22)^{*}$ & Instituto Nacional de Salud Pública: 1 \\
Web: $23(18)^{*}$ & Empresa privada: 1 \\
Sitios Web: $3(3)^{*}$ & \\
- Regulaciones en Investigación en Salud: $26(18)^{*}$ & \\
- Programas académicos de ética de investigación: $20(20)^{*}$ & \\
- Creación de nuevos comités de ética de & \\
investigación: $9(9)^{*}$ & \\
- Participación en Comités de Ética de & \\
Investigación: $28(28)^{*}$ & \\
- Participación en Comisiones Nacionales de & \\
Bioética: $6(6)^{*}$ & \\
\hline
\end{tabular}

${ }^{*}$ Se refiere al número de participantes del total. 


\section{Evaluación}

La evaluación ha sido necesaria para obtener información de lo aprendido y de la efectividad de la adquisición de habilidades. En la evaluación se mide: nivel de conocimiento mediante pruebas; habilidad para interactuar con los otros miembros del grupo y profesores en forma cualitativa, para diseñar investigación en bioética mediante la presentación de un proyecto y para reflexionar éticamente mediante la resolución de casos; además, se mide la participación en sesiones de comités de ética y habilidades de liderazgo para incluir lo aprendido en su trabajo profesional, mediante la presentación de un proyecto de intervención institucional. La eficacia del Programa se evalúa también por la efectividad con que los participantes realizan intervenciones en sus respectivas instituciones locales. La tabla 1 resume la contribución de los participantes.

Como forma de retroalimentación, los participantes evalúan el Programa cada año. La tabla 2 resume los aspectos importantes de las evaluaciones realizadas en los 10 años de experiencia en cuanto a contenidos, a docencia y a su eficacia.

Tabla 2. Evaluación del programa de ética de investigación biomédica y psicosocial por parte de los participantes.

\begin{tabular}{|c|c|c|}
\hline Aspectos positivos del programa & $\begin{array}{l}\text { Aspectos útiles para incorporar en las } \\
\text { instituciones }\end{array}$ & $\begin{array}{l}\text { Aspectos que necesitan ser } \\
\text { mejorados }\end{array}$ \\
\hline $\begin{array}{l}\text {-Calidad humana y profesional } \\
\text { de los académicos } \\
\text {-Actualidad de los contenidos } \\
\text {-Integración de los cursos } \\
\text {-Trabajo interdisciplinario y } \\
\text { transdisciplinario: interacciones } \\
\text { con profesionales y profesores de } \\
\text { distintas disciplinas, adquisición } \\
\text { de habilidades de comunicación } \\
\text { y de toma de decisiones } \\
\text {-Apoyo y logística } \\
\text { administrativa y coordinación } \\
\text { académica } \\
\text {-Completitud del programa y } \\
\text { carácter internacional } \\
\text {-Apoyo mediante beca } \\
\text {-Buen clima de aprendizaje: } \\
\text { compartir experiencias, trabajo } \\
\text { grupal, tutoría, actitud de } \\
\text { respeto } \\
\text {-Adquirir habilidades de } \\
\text { liderazgo } \\
\text {-Aprender a investigar en } \\
\text { Bioética } \\
\text {-Reconocimiento internacional: } \\
\text { Apoyo de Fogarty, OPS y la } \\
\text { Universidad de Chile } \\
\text {-Exigencia de continuidad y de } \\
\text { publicar }\end{array}$ & $\begin{array}{l}\text {-Adquisición de conocimientos para } \\
\text { introducir cursos y curricula de ética de } \\
\text { investigación en universidades } \\
\text {-Habilidades para la docencia en ética de } \\
\text { investigación } \\
\text {-Habilidades para diseńar proyectos de } \\
\text { investigación en bioética e investigaciones } \\
\text { en general incorporando requisitos éticos } \\
\text {-Aprendizaje de herramientas para } \\
\text { trabajar en forma interdisciplinaria y } \\
\text { transdisciplinaria } \\
\text {-Adquisición de un pensamiento crítico } \\
\text { para reflexionar en temas de ética de } \\
\text { investigación y cuestionar publicaciones } \\
\text { - Adquisición de habilidades para evaluar } \\
\text { ética y científicamente protocolos de } \\
\text { investigación en comités de ética } \\
\text {-Habilidades para escribir artículos en } \\
\text { temas de ética de investigación } \\
\text {-Mejoría de actitudes profesionales en } \\
\text { práctica clínica } \\
\text {-Conformación de redes } \\
\text {-Adquisición de habilidades en temas } \\
\text { de salud pública, prestando atención a } \\
\text { poblaciones vulnerables } \\
\text {-Adquisición de habilidades para usar } \\
\text { adecuadamente el consentimiento } \\
\text { informado en investigación } \\
\text {-Adquisición de habilidades para participar } \\
\text { en tomas de decisiones institucionales e } \\
\text { incorporar la bioética } \\
\text {-Adquisición de habilidades para escribir y } \\
\text { promover regulaciones en temas de ética de } \\
\text { investigación }\end{array}$ & $\begin{array}{l}\text {-Mayor uniformidad en los } \\
\text { candidatos en cuanto a previo } \\
\text { conocimiento en bioética } \\
\text {-Mayor acceso a documentación } \\
\text { escrita } \\
\text {-Incremento de experiencias } \\
\text { interdisciplinarias prácticas } \\
\text {-Obtención de un grado académico } \\
\text { de la universidad por participar en el } \\
\text { programa } \\
\text {-Mayor reflexión en temas de ética } \\
\text { de investigación psicosocial } \\
\text {-Mayor gerencia en resolver } \\
\text { problemas administrativos } \\
\text {-Mayor énfasis en evaluar los } \\
\text { resultados del aprendizaje } \\
\text {-Falta de tiempo para aprender en } \\
\text { mayor profundidad metodología } \\
\text { de investigación cualitativa y } \\
\text { cuantitativa } \\
\text {-Falta de tiempo para cumplir todos } \\
\text { los requisitos de investigación y } \\
\text { estudio } \\
\text { - Mayor apoyo de tutoría } \\
\text {-Mayor aplicación al contexto } \\
\text { latinoamericano en algunos de los } \\
\text { cursos } \\
\text {-Mayor trabajo autónomo } \\
\text { - Mayor aprendizaje en técnicas } \\
\text { de comunicación y habilidades de } \\
\text { trabajo interdisciplinario } \\
\text {-Mayor apoyo para realizar la } \\
\text { investigación } \\
\text { - Evitar repetir temáticas }\end{array}$ \\
\hline
\end{tabular}


Se observa que los aspectos bien evaluados del Programa coinciden con los objetivos de aprendizaje. Las propuestas de mejoría se han tratado de incorporar a lo largo de los años; no obstante, ha sido difícil conformar grupos homogéneos y poner completamente de acuerdo a los profesores en cuanto a la forma interdisciplinaria de trabajar.

\section{Conclusiones}

Aunque se han dado dificultades propias de las interacciones grupales, no completamente equilibradas en cuanto a formación previa en bioética y metodología de investigación de los miembros, la experiencia durante estos años ha sido fructífera. Con el apoyo del Programa, la mayoría de los participantes ha sido capaz de introducir cursos de ética de investigación en los currículos de programas de estudio universitarios, ha contribuido a formular regulaciones y ha participado activamente en comités de ética de investigación.

El aprendizaje grupal y poder concentrarse en reflexionar sobre temas de ética de investigación, apartándose de la rutina del trabajo profesional, muestran ser un sistema efectivo para adquirir habilidades y actitudes éticas e incorporarlas en las instituciones latinoamericanas.

\section{Referencias}

1. Lolas F, Rodríguez E. Ethics in psychosocial and biomedical research - A training experience at the Interdisciplinary Center for Bioethics (CIEB) of the University of Chile. Acta Bioethica 2012; 18(1): 9-56.

2. Filloux JC. Intersubjetividad y formación. Buenos Aires: Novedades Educativas; 1996.

3. Boud D, Cohen R, Sampson J. (eds.) Peer Learning in Higher Education. London: Kogan Page; 2001.

4. Zabalza MA. Competencias docentes del profesorado universitario. Calidad y desarrollo profesional. Madrid: Narcea; 2003: 126-127.

5. Hinman LM. Ethics: A pluralistic approach to moral theory. Belmont, CA: Wadsworth; 2004.

6. Ferrer JJ. La bioética como quehacer filosófico. Acta Bioethica 2009; 15(1):35-41. NIHMS132502.

7. Habermas J. Conciencia moral y acción comunicativa. Barcelona: Península; 1985.

Recibido: 24 de enero de 2012

Aceptado: 3 de febrero de 2012 